\title{
Quench Characteristics of a Two-Strand Superconducting Cable and the Influence of Its Length
}

\author{
G.B.J. Mulder, H.J.G. Krooshoop, L.J.M. van de Klundert, \\ University of Twente, Applied Superconductivity Centre \\ P.O.Box 217, 7500 AE Enschede, The Netherlands.
}

\author{
V.S. Vysotsky, \\ P.N. Lebedev Physical'Institute, \\ Leninskiy Prospect 53, Moscow 117333, USSR.
}

\begin{abstract}
The quench process of a multi-strand cable was investigated using the simplest system: two twisted wires. Several properties of the quench, such as the commutation of currents, the time scale, the resistance rate and the maximum voltage were determined experimentally or by calculation. Particular attention was given to the role of the cable length. Several samples with lengths varying from $1.5 \mathrm{~cm}$ to $12 \mathrm{~m}$ were made from an $A C$ superconductor with CuNi matrix. In the experiment, the decay of the currents is measured after initiating a local normal spot in one of the wires. An important conclusion is that the quench propagation and stability of a cable depends on its length and can therefore be influenced by soldering it at certain intervals.
\end{abstract}

\section{INTRODUCTION}

The application of superconductors for high-current switches or for AC purposes leads to a conductor design where several wires with a high-resistive matrix (of ten CuNi) are cabled either by twisting or by braiding. In order to reduce the $\mathrm{AC}$ losses, the adjacent wires in the cable should have poor electrical contact and they are therefore usually insulated. Such a type of cable has a very specific quench behaviour of which a general description has been given in $[1,2,3,4]$. The purpose of the present paper is to investigate in more detail the influence of the length of cable on the process.

Usually, it is impossible to obtain cryogenic or dynamic stabilization for the above-mentioned conductors because of the high resistivity of their matrix. Of course, the conductor can be adiabatically stable if thermal disturbances, for example due to wire movement, are kept below a safe level. A practical implication is that impregnation of the conductor is necessary. The combination of poor cooling and a high matrix resistivity leads to a very short "minimum propagation zone" (well below $1 \mathrm{~mm}$ at the usual current levels) and a "cold-end recovery current" which is almost zero $[5,6]$. So, in a cable with CuNi matrix, even a very small normal spot cannot disappear and will propagate throughout the wire until its current has dropped to almost zero. However, the current will be commutated to the other strands of the cable via the electrical contacts made at its ends. Therefore, under certain conditions it is nevertheless possible that a normal zone disappears without causing a quench of the whole cable.

Manuscript received June 24, 1991. These investigations have been supported by FOM, The Netherlands Foundation for Fundamental Research on Matter.
The conclusions of [1] can briefly be summarized as follows. Suppose a two-strand cable carries a transport current $I_{\text {tot }}$ and strand \#1 is locally heated using a short heat pulse of energy $E_{h}$, just enough to create a normal spot. Depending on $I_{\text {tot }}$ the following three cases can be distinguished:

a) Redistribution. The current in strand \#1 is fully commutated to $\# 2$, and $\# 2$ remains superconductive. So, the cable does not quench. Strand \#1 will recover after its current has nearly dropped to zero and then the current slowly diffuses from \#2 into \#1 again because the joint resistances impose a more or less homogeneous distribution of current over the two strands.

b) slow quench. If $I_{\text {tot }}$ exceeds a certain threshold level $I_{s}$, redistribution becomes impossible. Strand \#1 transfers its current to \#2, but strand \#2 cannot carry the excess current so the whole cable quenches. During the quench, the two normal zones become almost equal in length and they propagate in both directions at the usual velocity of the order of 10 to $100 \mathrm{~m} / \mathrm{s}$.

c) Fast quench. Above a second threshold level $I_{f}$, a new type of quench occurs. The essential aspect of this quench is that when strand \#2 becomes resistive due to the excess current of \#1, its resistance grows substantially faster than in \#1, so after some time the current of $\# 2$ expelled back to \#1. Then, \#1 quenches again. This quench-back effect repeats several times and the result is a purely electromagnetic phenomenon where the whole cable becomes resistive in a very short time at an apparent propagation velocity of the order of 10-100 kilometers per second.

The time associated with the commutation of current between strands and the amount of energy that is dissipated depends on the stray inductance between the strands, i.e. on the length of the cable. Therefore, it is expected that $I_{s}$ and $I_{f}$ also depend on the cable length. The threshold levels $I_{s}$ and $I_{f}$ are important for stability and protection reasons. So, one of the ideas we would like to test is whether the properties of the cable can be improved by soldering the cable at regular intervals of a certain length.

\section{I. THE EXPERIMENTAL SETUP}

A two-strand cable was twisted using two insulated $\mathrm{NbTi} / \mathrm{CuNi}$ conductors. The conductor has a bare diameter of $0.2 \mathrm{~mm}, 574 \mathrm{filaments}$ and a critical current of about $120 \mathrm{~A}$ under self field conditions. The cable was wound on a round bobbin as shown in Fig. 1. Then, the cable was soldered at certain positions lover a length of $15 \mathrm{~cm}$ ) to create several sections of different lengths. Each strand of each section was supplied with a smali heater made by winding a manganin wire around 
744

the strand over a length of about $0.5 \mathrm{~mm}$. A small piece of $0.13 \mathrm{~mm}$ Kapton foil prevents direct thermal contact of the heater with the other strand. Finally, the cable was impregnated with a thin layer of STYCAST 2850 FT epoxy and soldered to current leads using $15 \mathrm{~cm}$ long joints.

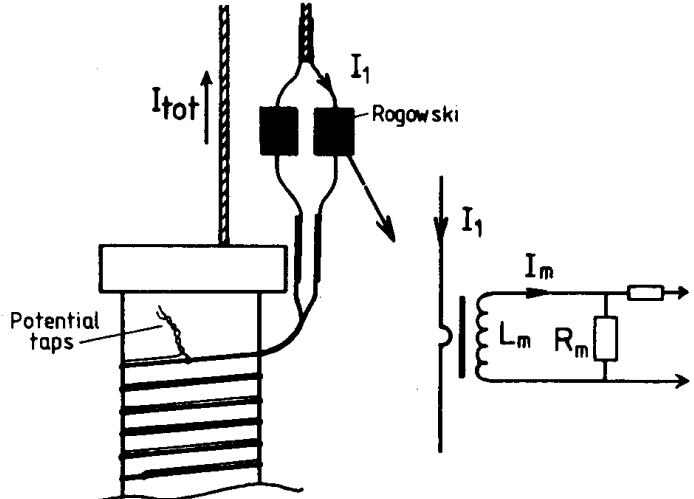

Fig. 1. Scheme of the experimental set-up, showing also the circuit of the self-integrating Rogowski.

One way to find out the parameters of the quench is to measure the resistive voltage of the cable. The potential wires should follow the cable in order to avoid the inductive component. During redistribution of current from \#1 to \#2, the voltage measured this way is equal to half the internal voltage $R_{1} I_{1}$. Much information can be obtained from this signal, such as the current and resistance as a function of time. However, if normal regions are present in both strands, interpretation of the voltage is more difficult. Therefore, to study the slow and fast quenches, we also used a second method where the currents were measured with two small Rogowski coils, as illustrated in Fig. 1. Passive RL integration of the Rogowski voltage is obtained with a low-ohmic load $R_{m}$. Consequently, for fast changing signals the current is measured directly instead of its derivative: The voltage over $R_{m}$ is $R_{m} I_{1} / N$ as long as $L_{m} \gg R_{m} \Delta t$ (except for the offset). In our case $R_{m}$ is $0.1 \Omega$ and $L_{m}$ is $5.5 \mathrm{mH}$ using 100 turns on a small iron ring core, so the method is applicable for processes that take place within $10 \mathrm{~ms}$. Note that the iron core does not introduce non-linearities since the ampereturns $\mathrm{I}_{1}$ and $-\mathrm{NI}_{\mathrm{m}}$ cancel the field change at the core.

\section{RESULTS FOR REDISTRIBUTION}

\section{A. Expected behaviour of $I(t)$ and $V(t)$ versus length}

The current decay during redistribution can be predicted using the model of [2]. In this model, the cable is replaced by two coupled RL-circuits that are connected to an external inductance $\mathrm{L}_{e}$ representing the wiring to the tested section. A further assumption is that the one-dimensional propagation velocity in a wire is a linear function of the current, i.e. $v_{p}=c I$. This is a rather good approximation for wires having a CuNi matrix, see Fig. 2. The current in the heated strand then satisfies

$$
I(t)=I_{0} / \cosh ^{2}\left(t \sqrt{\pi c I_{0} / L_{e f f}}\right) \text {, }
$$

where $I_{0}$ is the initial current in the strand, $n$ the resistance per meter and $\mathrm{L}_{\text {erf }}$ the effective inductance of the circuit enclosed by the two strands. $\mathrm{L}_{\text {eff }}$ is proportional to the length of cable [3] :

$$
\mathrm{L}_{\text {eff }} \cong \mathrm{L}_{1}+\mathrm{L}_{2}-2 \mathrm{M}_{12} \cong 4 \cdot 10^{-7} \ell(\ln (\mathrm{d} / \mathrm{a})+0.25) \text {, }
$$

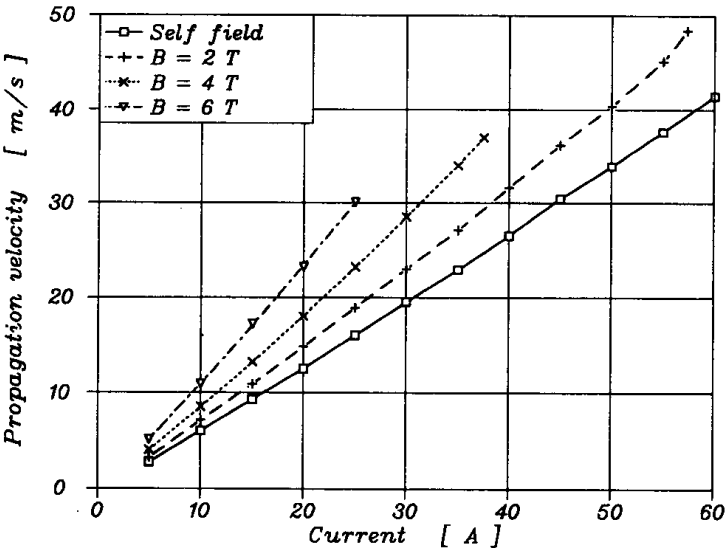

Fig. 2. Measured longitudinal normal zone velocity of the applied $\mathrm{NbTi} / \mathrm{CuNi}$ conductor for different values of the external field [6]

where $\ell$ is the cable length, $d$ the distance between the strands and a the radius of the filamentary area of the conductor.

The validity of (1) and (2) can be checked by measuring the voltage signal $V(t)$ over the cable during redistribution. The measured voltage. is half the internal inductive voltage, i.e. $-\frac{1}{2} \mathrm{~L}_{\text {eff }} \mathrm{I}$. So using (1) we get

$$
V(t)=I_{0} \sqrt{n c I_{0} L_{e f f}} \frac{\sinh \left(t \sqrt{n c I_{0} / L_{e f f}}\right)}{\cosh ^{3}\left(t \sqrt{n c I_{0} / L_{e f f}}\right)} \text {. }
$$

\section{B. Comparison of measured redistribution with theory}

First, the relevant parameters of the cable were determined: $r=9.9 \Omega / \mathrm{m}, \quad c=0.67 \mathrm{~m} / \mathrm{s} / \mathrm{A}, \quad a=0.09 \mathrm{~mm}$ and $d=0.24 \mathrm{~mm}$. Then, $V(t)$ was measured for various samples with lengths ranging from $1.5 \mathrm{~cm}$ to $12.3 \mathrm{~m}$. Each measurement was started with a $100 \mu$ s heater pulse, just sufficient to create a normal spot. The heater energy $E_{h}$ is $50 \mu \mathrm{J}$, but only part of it flows into the wire. The results are given in Fig. 3. To facilitate

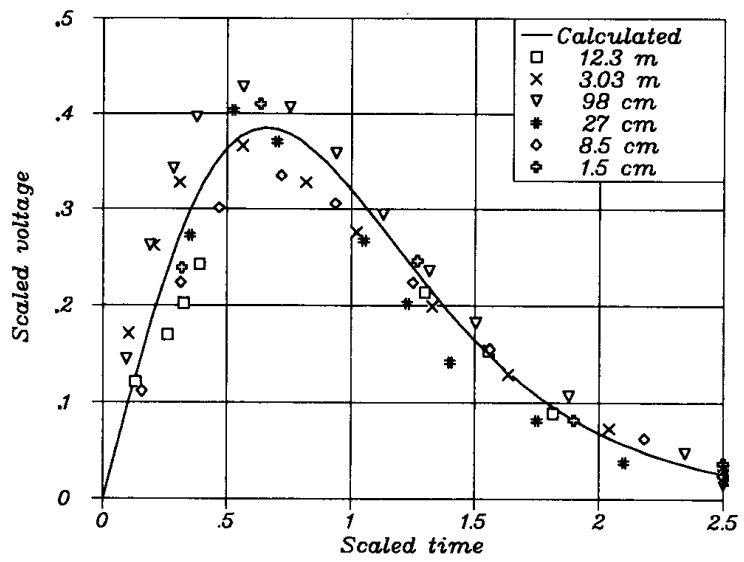

Fig. 3. Voltage signal during redistribution shown in dimensionless units, i.e. $V(t) /\left(I_{0} \sqrt{n c I_{0} L_{e f f}}\right)$ is plotted versus $t \sqrt{n c I_{O} / L_{e f f}}$. The points were measured and the solid line was calculated using eq. (3). 
comparison with the calculation, the results are presented in a scaled form, 1.e. $V(t) /\left(I_{0} \sqrt{r c I_{0} L_{e f f}}\right)$ is plotted versus $t \sqrt{r c I_{0} / L_{e f f}}$. The agreement is fairly good. In the experiment, the problem arises that one is not sure whether the current $I_{\text {tot }}$ is equally shared between the two strands at the start of a measurement. Later, it was found that the currents in the strands (under DC as well as AC conditions) sometimes differ $10 \%$ and this can easily explain the discrepancies seen in Fig. 3.

A measure for the time scale of the process is $\tau$, defined as the time measured in the $V(t)$ graph between the points where the voltage is $33 \%$ of the peak value. According to (3), the peak voltage is $2 / 9 \sqrt{3} \cdot \mathrm{I}_{0} \sqrt{\pi c I_{0} L_{\text {erf }}}$ and $\tau$ is $1.5 \sqrt{\mathrm{L}_{\text {eff }} /\left(\Omega \mathrm{CI} \mathrm{I}_{0}\right)}$. Figure 4 shows the calculated and measured $\boldsymbol{\tau}$ for different values of the sample length $\ell$ and the initial current $I_{0}$. The agreement is quite good except for one measurement corresponding to the $27 \mathrm{~cm}$ sample which is $30 \%$ too low. This demonstrates that the model and the parameters used in it are sufficiently accurate.

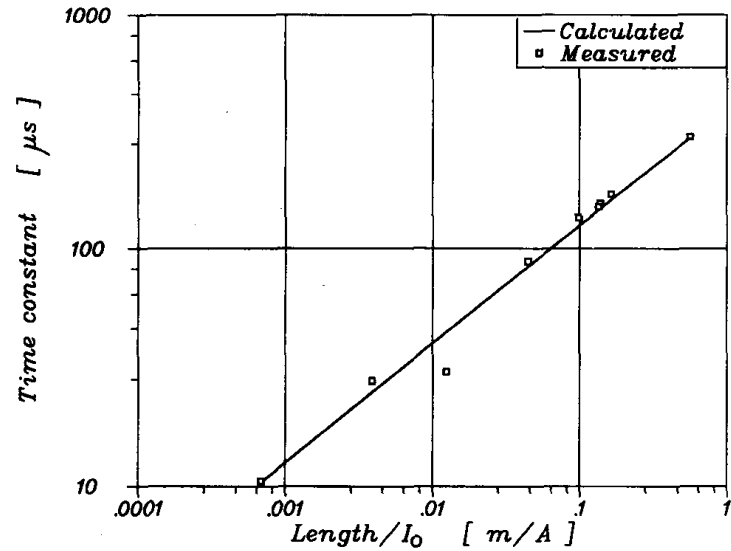

Fig. 4. Time $\tau$ of the redistribution process measured for various sample lengths and currents. The solid line represents the calculation.

\section{Resistance growth and dissipated energy}

The total length of the normal zone $\ell_{n}(t)$ can be found by integrating the propagation velocity

$$
\ell_{n}(t)=\int_{0}^{t} 2 c I d t=2 \sqrt{\frac{C I_{0} L_{e f f}}{n}} \tanh \left(t \sqrt{\frac{n C I}{L_{e f f}}}\right) \text {. }
$$

The final normal length is very short, only $6 \mathrm{~mm}$ for the $12.3 \mathrm{~m}$ sample. For the shortest sample, the normal length is of the order of the diameter of the wire so the one-dimensionality of the problem is questionable, but nevertheless eqs. (1) and (3) still give a good description of the experimental results. The dissipated energy in the normal zone during the redistribution process is $1 / 2 L_{\text {erf }} I_{0}^{2}$. For example, if $22 \mathrm{~A}$ is redistributed in the $12.3 \mathrm{~m}$ sample, $1.5 \mathrm{~mJ}$ is dissipated and under adiabatic conditions it would cause a temperature rise of about $40 \mathrm{~K}$. The dissipated energy is cooled away from the hot spot in all directions, especially in longitudinal direction because of the relatively high thermal conductivity of the conductor compared with the epoxy and insulation material. Therefore, only a small fraction of the heat enters the adjacent strand.

\section{RESULTS FOR FAST AND SLOW QUENCHES}

\section{A. Determination of the threshold levels $I_{s}$ and $I_{f}$}

To study the slow and fast quenches, an additional number of samples were prepared having lengths $3.8 \mathrm{~cm}$, $8.7 \mathrm{~cm}, 30 \mathrm{~cm}, 1.0 \mathrm{~m}, 3.0 \mathrm{~m}$ and $8.0 \mathrm{~m}$. The difference between redistribution, slow quench and fast quench can be demonstrated by plotting the peak resistive voltage over the cable as a function of the initial current.

Fig. 5 shows such a plot for the 1,3 and $8 \mathrm{~m}$ samples. The presence of two threshold levels $I_{s}$ and $I_{f}$ is very clear, because the peak voltage suddenly increases by a factor of 10-100 if $I_{\text {tot }}$ is only slightly increased. This difference, however, becomes less pronounced as the sample length decreases. It is difficult to make the distinction between slow or fast quench for samples shorter than $10 \mathrm{~cm}$. Of course, the peak. voltage is an indication for the speed of the process. In the case of a fast quench the full length of cable becomes resistive in less than $100 \mu \mathrm{s}$. Then, the current decays exponentially with a time constant given by total self inductance of the sample including wiring (about $150 \mu \mathrm{H}$ in our experiment) and the full resistance of the twostrand cable $(r / / 2)$. It can be concluded from the measured voltage signals that the fast quench stops at a soldered section. The normal zones propagate through the soldered section only at a relatively low velocity.

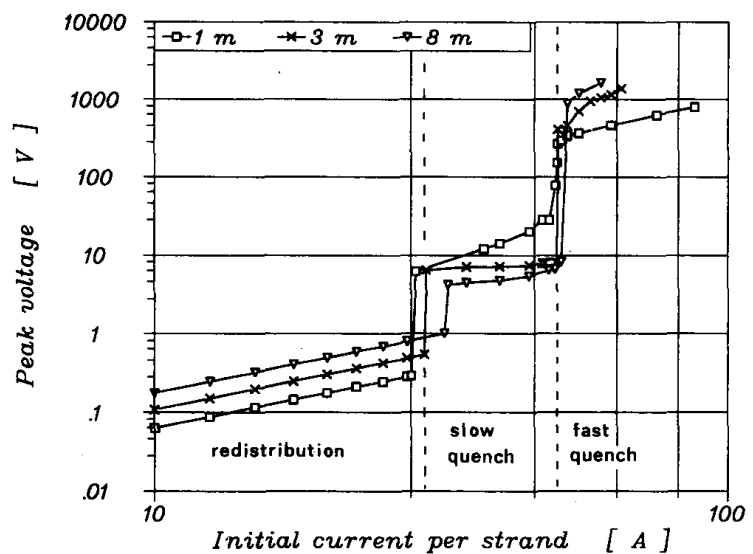

Fig. 5. Measured peak voltage versus initial current per strand $I_{0}$, for different lengths of cable.

The dependence of $I_{s}$ and $I_{f}$ on the sample length is shown in Fig. 6. The scattering of points in Fig. 6 illustrates the poor reproducibility of the measurement of $I_{f}$, but the expected increase of $I_{f}$ with increasing length can still be recognized [3]. The measurements of I reproduce better. In the measured range, $I$ is also an increasing function of $\ell$. A likely explanation for the decrease of $I_{s}$ for short samples is that the high current rates during the redistribution affect the stability of the strand, as pointed out by Mints [7]. From (4), the maximum current rate can be calculated as $0.8 \cdot \mathrm{I}_{0} \sqrt{\mathrm{rcI} \mathrm{I}_{0} / \mathrm{L}_{\text {eff }}}$, which amounts to well over $10^{6} \mathrm{~A} / \mathrm{s}$ for the shortest samples. This means that during the current commutation, a strand generates a magnetic field change at its neighbour of more than $1000 \mathrm{~T} / \mathrm{s}$ ! 


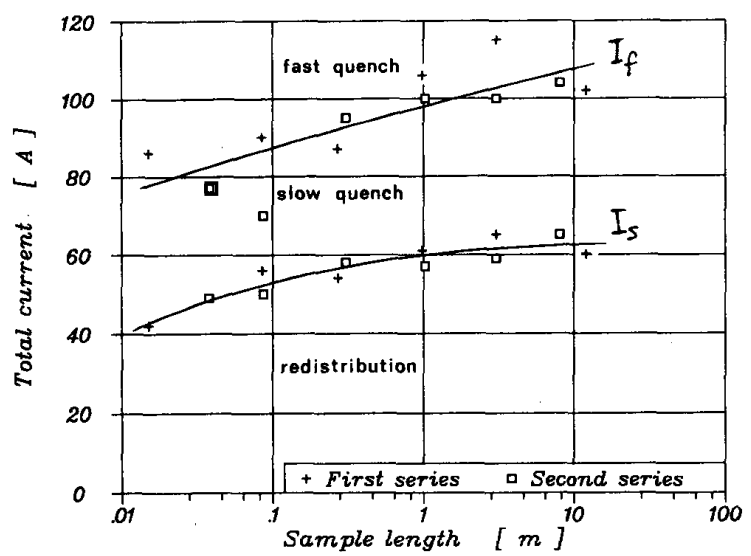

Fig. 6. Threshold currents $I_{s}$ and $I_{f}$ as a function of the sample length.

\section{B. Thermal contact between neighbours}

It was useful to find out how much time is required for a normal spot in one strand to create a normal spot in its nelghbour. The following experiment was carried out. The two strands of the $3 \mathrm{~m}$ sample were connected to separate power supplies and supplied with different DC currents. Then, after heating strand \#1, the time delay between the appearance of resistive voltages in $\# 1$ and \#2 was measured. The currents $I_{1}$ and $I_{2}$ are almost constant during the first millisecond of the experiment, so actually the length of the sample is unimportant here. The results are presented in Fig. 7 for different values of $I_{1}$ and $I_{2}$. It can be concluded that the time required for thermal conduction between adjacent strands is much longer than the duration of the fast quench process, but it is comparable to the duration of a slow quench.

The duration of fast quenches is so short that the thermal conduction between neighbouring strands can be neglected and only the magnetic coupling between the strands is relevant. For slow quenches or redistribution the thermal contact between the strands should be considered. It is expected, for example, that a better thermal contact will lead to a reduction of $I_{s}$. The total energy that is dissipated during the redistribution process increases Iinearly with $\ell$, and the dissipated energy per unit length (in the hot spot) is $2 / 3 I_{0} \sqrt{\pi I_{0} L_{\text {eff }} / C}$, i.e. proportional to $\sqrt{\ell}$. Consequently, the temperature rise of a strand caused by the heat of a normal region in its neighbour becomes increasingly important for large $\ell$. This should lead to a reduction of $I_{s}$ for long samples, but such a reduction is not observed in the measured curve of Fig. 6. Therefore, an interesting future experiment is to investigate samples that are significantly lorger than $12 \mathrm{~m}$.

\section{v. CONCLUSIONS}

In order to get a better understanding of the complex quench processes occurring in multi-strand cables with insulated strands, it is useful to start by character-

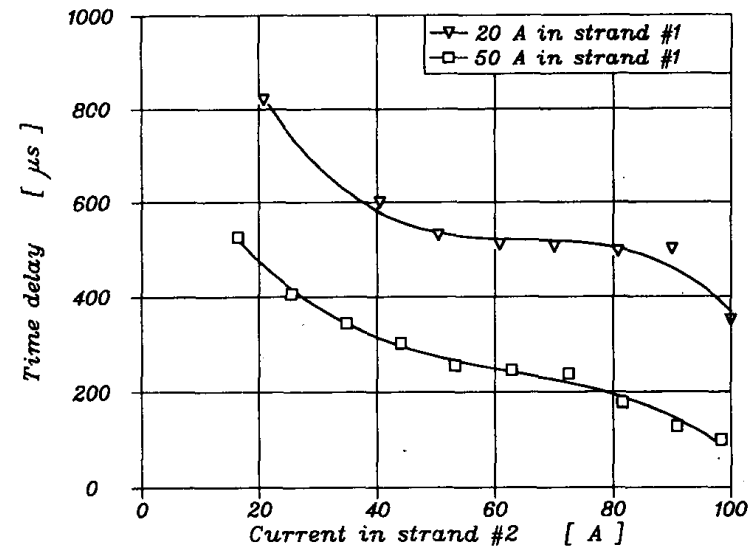

Fig. 7. Measured time for a normal spot to propagate between adjacent strands. The experiment was carried out with the $3 \mathrm{~m}$ sample.

izing the two-strand case. It was demonstrated that the length of such a cable plays an important role in the quench behaviour. The effect of the cable length on the threshold currents $I_{s}$ and $I_{f}$ has been established experimentally for lengths between $1.5 \mathrm{~cm}$ and $12 \mathrm{~m}$. For protection or switching purposes it would be interesting to lower the value of $I_{f}$. From a stability point of view it is important that $\mathrm{I}_{\mathbf{s}}$ is as large as possible. our conclusion is that such properties of the cable can in principle be improved by changing the geometry of the cable or its length.

\section{ACKNOWLEDGMENT}

The authors would like to thank G. Rohaan who carried out a large part of the measurements.

\section{REFERENCES}

[1] V.S. Vysotsky, V.N. rsikhon, and G.B.J. Mulder, "Quench development in superconducting cable having insulated strands with high resistive matrix (part 1, experiment)," this conference.

[2] G.B.J. Mulder, V.S. Vysotsky and LJ.M. van de Klundert, "Quench development in superconducting cable having insulated strands with high resistive matrix (part 2, analysis)," this conference.

[3] M. Iwakuma et al., "Abnormal quench process with very fast elongation of normal zone in multi-strand superconducting cables," Cryogenics, vol. 30, 1990, pp. 686-692.

[4] G.B.J. Mulder, H.J.G. Krooshoop, A. Nijhuis, H.H.J. ten Kate and L.J.M. van de Klundert, "A study of quench current and stability of high-current multi-strand cables having a $\mathrm{Cu}$ or a CuNi matrix," Adv. in Cryog. Eng., vol. 36A, 1990, pp. 279-286.

[5] M.N. Wilson, "Superconducting magnets," Clarendon Press, Oxford, 1983.

[6] H.H.J. ten Kate, H. Boschman, and L.J.M. van de Klundert, "Normal zone propagation velocities in superconducting wires having a high-resistivity matrix," Adv. in Cryog. Eng., vol. 34, 1988, pp. 1049-1056.

[7] R.G. Mints, A.L. Rakhmanov, "The current-carrying capacity of twisted multifilamentary superconducting composites," J. Phys. D: Appl. Phys., vol. 20, 1988, pp. 826-830. 